\title{
Computer Determination of Contact Stress Distribution and Size of Weight Bearing Area in the Human Hip Joint
}

\author{
ALEŠ IGLIČ $\check{\mathrm{a}}^{\mathrm{a}, *}$, V. KRALJ-IGLIČ ${ }^{\mathrm{b}}$, M. DANIEL ${ }^{\mathrm{a}, \mathrm{c}}$ and A. MAČEK-LEBAR ${ }^{\mathrm{a}}$ \\ ${ }^{\mathrm{a}}$ Faculty of Electrical Engineering, Tržaška 25, Ljubljana SI-1000, Slovenia; ${ }^{\mathrm{b}}$ Department of Orthopaedic Surgery and Institute of Biophysics, Faculty of \\ Medicine, Lipičeva 2, Ljubljana SI-1000, Slovenia; ${ }^{\mathrm{c}}$ Faculty of Mechanical Engineering, Tehnicka 4, 166-07 Praha 6, Czech Republic
}

(Received 3 August 2001; In final form 10 January 2002)

\begin{abstract}
The mathematical models and the corresponding computer program for determination of the hip joint contact force, the contact stress distribution, and the size of the weight bearing area from a standard anteroposterior radiograph are described. The described method can be applied in clinical practice to predict an optimal stress distribution after different operative interventions in the hip joint and to analyze the short and long term outcome of the treatment of various pathological conditions in the hip. A group of dysplastic hips and a group of normal hips were examined, with respect to the peak contact stress normalized by the body weight, and with respect to the functional angle of the weight bearing area. It is shown that both these parameters can be used in the assessment of hip dysplasia.
\end{abstract}

Keywords: Hip joint; Computer simulation; Contact stress distribution; Radiograph

\section{INTRODUCTION}

The hip joint is a multiaxial spheroidal joint where the articulating bone surfaces are covered with articular cartilage. A synovial membrane underlying the interior of the joint capsule secretes lubricant called synovial fluid [1]. Due to synovial fluid, the tangential stress in the hip joint articular surface is negligible compared to the normal stress [2]. In the following, the term hip joint contact stress is used for the normal stress in the hip joint articular surface.

It was indicated that an excessive hip joint contact stress is an important factor accelerating degenerative processes in the hip joint [3-5]. Also, it was found that the peak stress in the hip joint is higher in women than in men [6]. As women have a higher incidence of arthrosis, these results favor the hypothesis that elevated stress in the hip joint (due to characteristic femoral and pelvic shape) can be one of the reasons for greater incidence of arthrosis in the female population [7].

In this work, we present a computer method for estimation of peak contact stress and of the size of the weight bearing area in human hip joint in one-legged stance body position by using a standard anteroposterior roentgenograph. The contact stress distribution in the hip joint is determined in two steps. First, the hip joint resultant force $\mathbf{R}$ is determined by solving the equilibrium equations for forces and torques in the one-legged stance where the individual variations in the femoral and pelvic geometry are determined from standard anteroposterior roentgenographs. Second, the magnitude and direction of $\mathbf{R}$ are used as the input data into the model for determination of stress distribution. For that, centre-edge angle $\left(\vartheta_{\mathrm{CE}}\right)$ and the radius of the femoral head $(r)$ should also be known. Two specific examples of application of the described computer program for determination of the contact stress distribution and the size of the weight bearing area in the human hip joint are presented: the peak contact stress in different pelvic shapes is calculated and the average peak contact stress and the average weight bearing area are estimated for the group of normal hips and for the group of dysplastic [8] hips.

\section{DETERMINATION OF THE HIP JOINT RESULTANT FORCE FROM THE STANDARD ANTEROPOSTERIOR RADIOGRAPHS IN ONE- LEGGED STANCE}

\section{Equations of Static Equilibrium of the Body in the One-legged Stance}

The origin of the Cartesian coordinate system is chosen in the femoral head center of the loaded leg so that the $x$ and $z$

*Corresponding author. Tel.: +386-1-4264-630. Fax: +386-1-4768-235. E-mail: ales.iglic@fe.uni-1j.si. 


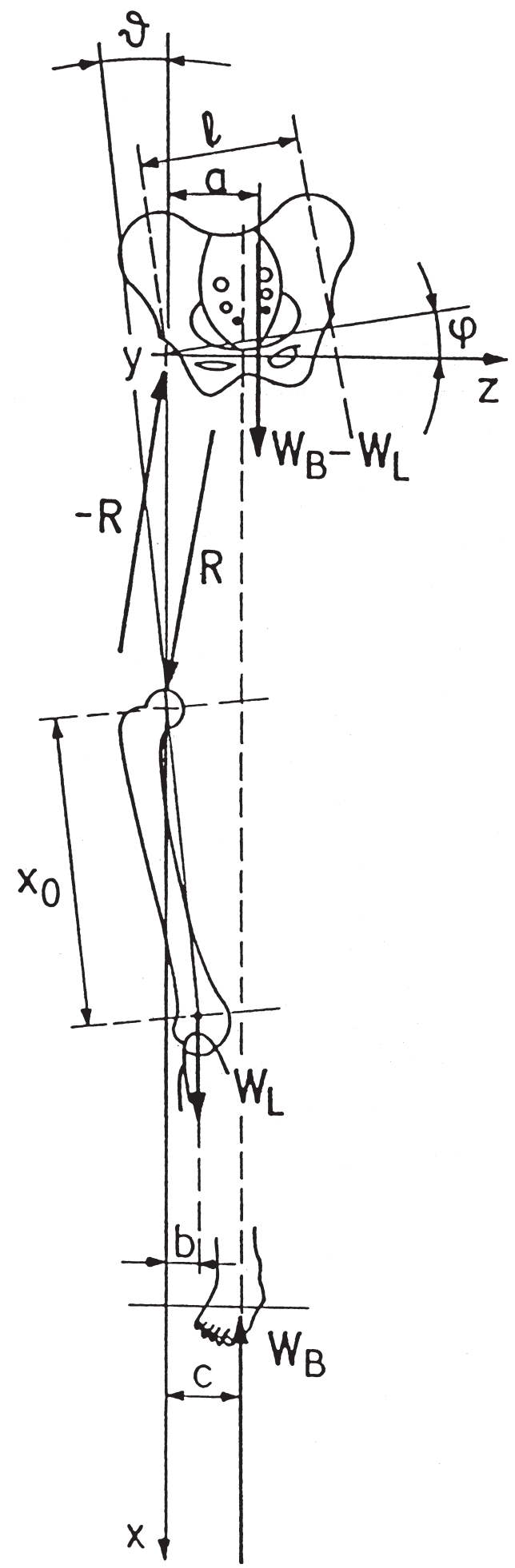

FIGURE 1 The characteristic forces, moment arms and geometrical parameters of the described model of the hip in one-legged stance body position.

axes lie in the frontal plane while $y$ axis points in the posterior direction (Fig. 1). Due to simplicity, the body is divided into two segments. The first segment is the loaded leg and the second segment is the rest of the body. In the one-legged stance (Fig. 1), the second segment bears the partial body weight $\left(\mathbf{W}_{\mathrm{B}}-\mathbf{W}_{\mathrm{L}}\right)$, where $\mathbf{W}_{\mathrm{B}}$ is the body
TABLE I The relative cross-sectional areas of the model muscles

\begin{tabular}{lccc}
\hline Muscle & $i$ & Group & $A_{i}$ \\
\hline Gluteus medius-anterior & 1 & $\alpha$ & 0.266 \\
Gluteus minimus-anterior & 2 & $\alpha$ & 0.113 \\
Tensor fasciae latae & 3 & $\alpha$ & 0.120 \\
Rectus femoris & 4 & $\alpha$ & 0.400 \\
Gluteus medius-middle & 5 & $\beta$ & 0.266 \\
Gluteus minimus-middle & 6 & $\beta$ & 0.113 \\
Gluteus medius-posterior & 7 & $\gamma$ & 0.266 \\
Gluteus minimus-posterior & 8 & $\gamma$ & 0.133 \\
Piriformis & 9 & $\gamma$ & 0.100 \\
\hline
\end{tabular}

weight and $\mathbf{W}_{\mathrm{L}}$ the weight of the loaded leg. The force and moment equilibrium equations for the second segment can be written in the form [9]:

$$
\begin{gathered}
\sum_{i} \mathbf{F}_{i}-\mathbf{R}+\left(\mathbf{W}_{\mathrm{B}}-\mathbf{W}_{\mathrm{L}}\right)=0, \\
\sum_{i}\left(\mathbf{r}_{i} \times \mathbf{F}_{i}\right)+\mathbf{a} \times\left(\mathbf{W}_{\mathrm{B}}-\mathbf{W}_{\mathrm{L}}\right)=0,
\end{gathered}
$$

where $\mathbf{a}=(0,0, a)$ is the moment arm of the force $\left(\mathbf{W}_{\mathrm{B}}-\right.$ $\left.\mathbf{W}_{\mathrm{L}}\right), \mathbf{r}_{i}$ is the radius vector of the $i$-th muscle force $\left(\mathbf{F}_{i}\right)$ application point drawn from the origin of the coordinate system to the muscle origin point on the pelvis. The model includes nine effective muscles, which are classified in three groups according to their positions: anterior $(\alpha)$, middle $(\beta)$ and posterior $(\gamma)$ (Table I). Each muscle is considered to act along straight line connecting the point of attachment on the pelvis (determined by the radius vector $\mathbf{r}_{i}$ ) and the point of attachment on the femur (determined by the radius vector $\mathbf{r}_{i}^{\prime}$ ). The rotation of the pelvis in the frontal plane around $y$-axis is described by the angle $\varphi$ while the rotation of the femur around $y$-axis in the frontal plane is described by the angle $\vartheta$ (Fig. 1). For $\vartheta=0$ and $\varphi=0$, the reference three-dimensional coordinates of the radius vectors $\mathbf{r}_{i}=\left(x_{i}, y_{i}, z_{i}\right)$ and $\mathbf{r}_{i}^{\prime}=$ $\left(x_{i}^{\prime}, y_{i}^{\prime}, z_{i}^{\prime}\right)$ are taken from Dostal and Andrews [10]. In general, the angles $\vartheta$ and $\varphi$ are not zero (Fig. 1), therefore, the reference coordinates of the muscle attachment should be corrected using the corresponding rotation matrixes. In this work, we take $\varphi=0$ and $\vartheta=\arcsin \left(b / x_{o}\right)$ where $x_{o}=42.3 \mathrm{~cm} \mathrm{[10]} \mathrm{is} \mathrm{the} \mathrm{length} \mathrm{of} \mathrm{the} \mathrm{femur} \mathrm{and} b$ is the $z$ coordinate of the moment arm of the force $\mathbf{W}_{\mathrm{L}}$ (Fig. 1).

The force of each individual muscle included in the model is written as [9]:

$$
\mathbf{F}_{i}=\sigma_{i} A_{i} \mathbf{e}_{i}
$$

where $A_{i}$ is the relative cross-sectional area of the $i$-th muscle (Table I) determined from the data of Johnston et al. [11], $\sigma_{i}$ is the average tension in the $i$-th muscle and $\mathbf{e}_{i}=\left(e_{i x}, e_{i y}, e_{i z}\right)$ is the unit vector in the direction of the force of the $i$-th muscle $(i=1,2, \ldots, 9)$ :

$$
\mathbf{e}_{i}=\frac{\mathbf{r}_{i}^{\prime}-\mathbf{r}_{\mathrm{i}}}{\left|\mathbf{r}_{i}^{\prime}-\mathbf{r}_{i}\right|}
$$


TABLE II The predicted values of the average muscle tensions $\left(\sigma_{i}\right)$ and the magnitude of the hip joint resultant force $(R)$ determined by the optimization method (second column) and by the reduction method (third column) and for $\ell=16.9 \mathrm{~cm}$ and $W_{\mathrm{B}}=800 \mathrm{~N}$.

\begin{tabular}{lcc}
\hline Muscle $(i)$ & $\begin{array}{c}\text { Optimization } \\
\sigma_{i}\left(\mathrm{~N} / \mathrm{cm}^{2}\right)\end{array}$ & $\begin{array}{c}\text { Reduction } \\
\sigma_{i}\left(\mathrm{~N} / \mathrm{cm}^{2}\right)\end{array}$ \\
\hline 1 & 111.9 & 36.2 \\
2 & 39.8 & 36.2 \\
3 & 34.5 & 36.2 \\
4 & 48.1 & 36.1 \\
5 & 102.7 & 191.0 \\
6 & 37.6 & 191.0 \\
7 & 78.4 & 14.6 \\
8 & 28.9 & 14.6 \\
9 & 15.0 & 14.6 \\
$\mathbf{R} / W_{\mathrm{B}}$ & 2.370 & 2.383 \\
\hline
\end{tabular}

The magnitude of the moment arm of the force $\left(\mathbf{W}_{\mathrm{B}}-\right.$ $\mathbf{W}_{\mathrm{L}}$ ) is determined from the $y$-component of the moment equilibrium equations for the first and the second body segment:

$$
\begin{gathered}
-W_{\mathrm{B}} c+W_{\mathrm{L}} b-M_{Y}=0, \\
\left(W_{\mathrm{B}}-W_{\mathrm{L}}\right) a+M_{Y}=0,
\end{gathered}
$$

where $a$ is $z$-coordinate of the moment arm $\mathbf{a}=(0,0, a), c$ is $z$-coordinate of the moment arm of the ground reaction force $-\mathbf{W}_{\mathrm{B}}$ (Fig. 1) and $M_{Y}$ is $z$-component of intersegmental moment $\mathbf{M}=\sum_{i}\left(\mathbf{r}_{i} \times \mathbf{F}_{i}\right)$. It follows from Eqs. (5) and (6) [12]:

$$
a=\frac{W_{\mathrm{B}} c-W_{\mathrm{L}} b}{W_{\mathrm{B}}-W_{\mathrm{L}}} .
$$

The moment arms $b$ and $c$ are expressed by the interhip distance $\ell: b=0.24 \ell, c=0.5 \ell$ [12]. The weight of the leg is approximated by the equation $W_{\mathrm{L}}=0.16 W_{\mathrm{B}}$ [13].

The described mathematical model for the body in the one-legged stance static position has six scalar equations given by three components of the vector equilibrium equations (Eqs. (1) and (2)), and 12 unknowns (three components of the force $\mathbf{R}=\left(R_{X}, R_{Y}, R_{Z}\right)$ and nine unknown muscle tensions $\sigma_{i}$ ). The number of unknowns of the model thus exceeds the number of model equations, therefore the problem is indeterminate and in general an infinite number of solutions satisfy the system of Eqs. (1) and (2). The problem can be solved either by reducing the number of the model unknowns (reduction method) or by taking into account additional optimization criteria for the muscles' actions (optimization method).

\section{Reduction Method}

The aim of the reduction method [9] is to modify an initially indeterminate problem to a determinate one by reducing the number of unknowns. For this purpose, the average tensions $\left(\sigma_{i}\right)$ in the particular muscle group are assumed to be equal: $\sigma_{1}=\sigma_{2}=\sigma_{3}=\sigma_{4}=\sigma_{\alpha}, \sigma_{5}=$ $\sigma_{6}=\sigma_{\beta}, \sigma_{7}=\sigma_{8}=\sigma_{9} \equiv \sigma_{\gamma}$. The equilibrium equations (Eqs. (1) and (2)) can be then solved and the unknown quantities $R_{X}, R_{Y}, R_{Z}, \sigma_{\alpha}, \sigma_{\beta}$ and $\sigma_{\gamma}$ are obtained (Table
II). The calculated $\mathbf{R}$ turned out to be lying nearly in the frontal plane of the body and its sagittal component did not exceed $1 \%$ of the frontal component in one-legged stance [9].

\section{Optimization Method}

Another procedure used to solve an initially indeterminate problem (Eqs. (1) and (2)) is the optimization method $[11,14]$. In this study, we select the nonlinear optimization criteria of minimal possible magnitude of the hip joint contact force [15], i.e. the criteria of the minimal possible bone loading. Due to simplicity, we are searching for the minimum of the square of magnitude of the hip joint reaction force:

$$
\begin{aligned}
F= & \left(\sum_{i=1}^{9} \sigma_{i} A_{i} e_{i x}+\left(W_{\mathrm{B}}-W_{\mathrm{L}}\right)\right)^{2} \\
& +\left(\sum_{i=1}^{9} \sigma_{i} A_{i} e_{i y}\right)^{2}+\left(\sum_{i=1}^{9} \sigma_{i} A_{i} e_{i z}\right)^{2},
\end{aligned}
$$

subject to three constraints (Eq. (2)):

$$
\begin{gathered}
G_{1}=\sum_{i=1}^{9}\left(Y_{i} \sigma_{i} A_{i} e_{i z}-z_{i} \sigma_{i} A_{i} e_{i y}\right)=0 \\
G_{2}=\sum_{i=1}^{9}\left(z_{i} \sigma_{i} A_{i} e_{i x}-x_{i} \sigma_{i} A_{i} e_{i z}\right)+a\left(W_{\mathrm{B}}-W_{\mathrm{L}}\right)=0 \\
G_{3}=\sum_{i=1}^{9}\left(x_{i} \sigma_{i} A_{i} e_{i y}-y_{i} \sigma_{i} A_{i} e_{i x}\right)=0
\end{gathered}
$$

In order to solve the described optimization problem, we used the constrained Fletcher-Powell algorithm [16] where a modified objective function is defined as:

$$
\Phi=F-\sum_{k=1}^{3} \lambda_{k} G_{k}+B \sum_{k=1}^{3} G_{k}^{2}
$$

where $\lambda_{k}$ and $B$ are parameters. When the convergence is achieved $G_{k}=0$ and $F=\Phi$. The values of $\sigma_{i}$ and $R$ determined by this optimization technique are given in Table II. It can be seen in Table II that the calculated values of $\sigma_{i}$ by using the reduction method and by using the optimization technique are considerably different. However, the predicted values of $R / W_{\mathrm{B}}$ do not differ that much. Since in this work, we are interested only in the hip joint resultant force, in the following $\mathbf{R}$ is calculated using the simple reduction method.

\section{Determination of the Hip Joint Resultant Force for an Individual Patient Using the Standard Anteroposterior Radiograph}

The calculated hip joint resultant force $\mathbf{R}$ should be scaled by the individual variations in femoral and pelvic 
geometry $[6,17,18]$. For this reason, the reference values of the model muscle attachment points [10] and the interhip distance $\ell$ are rescaled in order to adjust the configuration of the hip and pelvis for the individual person. Thereby, the following values of the hip and pelvic geometrical parameters from the standard anteroposterior roentgenograph of a given patient are measured (Fig. 2): the interhip distance $(\ell)$, the pelvic height $(H)$, the pelvic width $(C)$, the vertical and the horizontal distance from the center of the femoral head to the effective muscle attachment point $(T)$ on the greater trochanter $(z$ and $x$, respectively). The point $T$ is determined by the intersection of the contour of the greater trochanter and the normal through the midpoint of the straight line connecting the most lateral point (point 1) and the highest point (point 2) on the greater trochanter $[17,18]$. The above-described geometrical parameters are used to scale the respective reference values of the attachment points of the muscles included in the model (Table I) and the interhip distance for an individual person. The reference values of $H, C, \ell, z$ and $x$ are adopted from Dostal and Andrews [10] and Kersnič et al. [7]. While taking the roentgenograph, both femurs should be in the zero joint configuration where the straight line connecting the femoral head center and the midpoint between the lateral and medial epicondyle is perpendicular to the straight line through both femoral head centers [10]. The abduction or adduction of the legs from this reference configuration would affect the accuracy of the correct values of the coordinates of the point $T$, i.e. $z$ and $x$.

\section{DETERMINATION OF THE CONTACT STRESS DISTRIBUTION AND THE FUNCTIONAL ANGLE OF THE WEIGHT BEARING AREA OF THE HIP JOINT}

As it is mentioned above, the input parameters of the mathematical model for calculation of the hip stress distribution and for calculation of the size of the weight bearing area in the hip joint are the magnitude of the resultant hip force $\mathbf{R}$, the direction of $\mathbf{R}$ represented by its inclination with respect to the sagittal plane $\left(\vartheta_{\mathrm{R}}\right)$, the center edge angle $\vartheta_{\mathrm{CE}}$ and the radius of the femoral head $r$ (Fig. 2). The hip joint resultant force $\mathbf{R}$ is considered to be lying in the frontal plane.

Within the model of stress distribution used in this work [19], it is assumed that when unloaded, the acetabular shell and the femoral head have spherical shape with coincident centers. Upon loading, the intermittent cartilage layer is squeezed. The contact hip stress at any point of the weight-bearing area $(p)$ is proposed to be proportional to strain in the cartilage layer. The point of closest approach of the spherical surfaces of the acetabulum and the femoral head is called the stress pole with the spherical coordinates $\Theta$ and $\Phi$ [19]. The polar angle $\Theta$ determines the angular displacement of the pole from the vertical axis, while the azimuthal angle $\Phi$

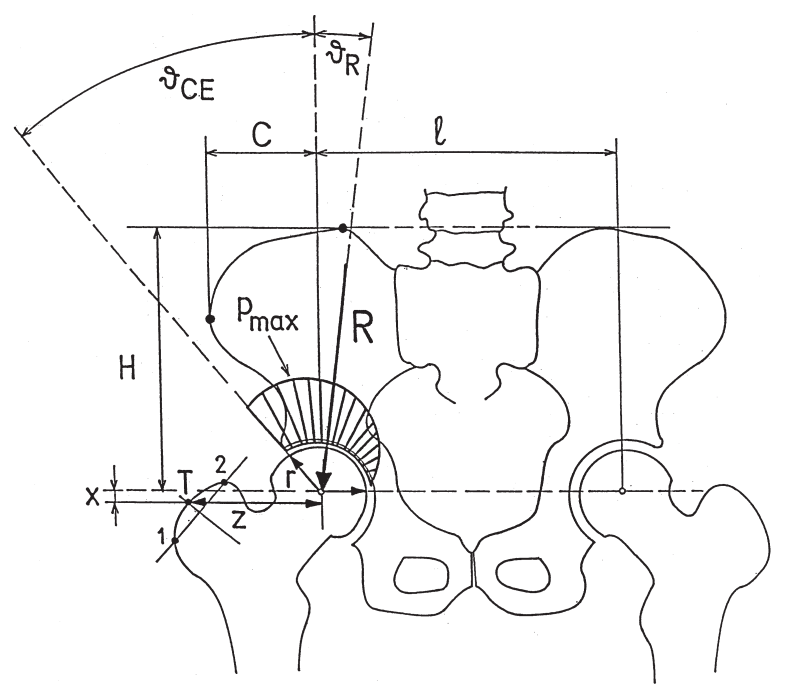

FIGURE 2 The geometrical parameters of the hip and pelvis needed for determination of the hip joint resultant force and contact stress distribution in the hip joint. The stress distribution and the resultant hip joint force $\mathbf{R}$ are also shown schematically. Symbol $\vartheta_{\mathrm{R}}$ denotes the inclination of $\mathbf{R}$ with respect to the vertical.

describes the angular displacement of the pole in the horizontal plane from the frontal plane in the counterclockwise direction. The above assumptions lead to the cosine dependency of the contact stress distribution in the hip joint $[19,20]$ :

$$
p=p_{o} \cos \gamma
$$

where $p_{o}$ is the value of stress in the pole and $\gamma$ is the angle between a given point and the stress pole. The lateral border of the weight-bearing area is determined by the acetabular geometry, while the medial border is defined as the curve where stress vanishes, i.e. where $\cos \gamma=0$. With known magnitude and direction of $\mathbf{R}$, the distribution of the contact stress in the hip joint can be computed from the equation

$$
\int_{S} p \mathrm{~d} \mathbf{S}=\mathbf{R}
$$

where we integrate over the weight-bearing area. The solution of the system of Eqs. (13) and (14) [19] yields (for the case when $\mathbf{R}$ lies in the frontal plane and when pole lies on the lateral side of the acetabular contact hemisphere or outside the acetabular contact hemisphere in the lateral direction) the spherical coordinate of the stress pole $(\Theta)$ and the value of stress at the pole $\left(p_{o}\right)$ :

$$
\tan \left(\vartheta_{\mathrm{R}}+\Theta\right)=\frac{\cos ^{2}\left(\vartheta_{\mathrm{CE}}-\Theta\right)}{\frac{\pi}{2}+\vartheta_{\mathrm{CE}}-\Theta+\sin \left(\vartheta_{\mathrm{CE}}-\Theta\right) \cos \left(\vartheta_{\mathrm{CE}}-\Theta\right)}
$$

$$
p_{o}=\frac{3 R \cos \left(\vartheta_{\mathrm{CE}}+\Theta\right)}{2 r^{2}\left(\frac{\pi}{2}+\vartheta_{\mathrm{CE}}-\Theta+\sin \left(\vartheta_{\mathrm{CE}}-\Theta\right) \cos \left(\vartheta_{\mathrm{CE}}-\Theta\right)\right)} .
$$




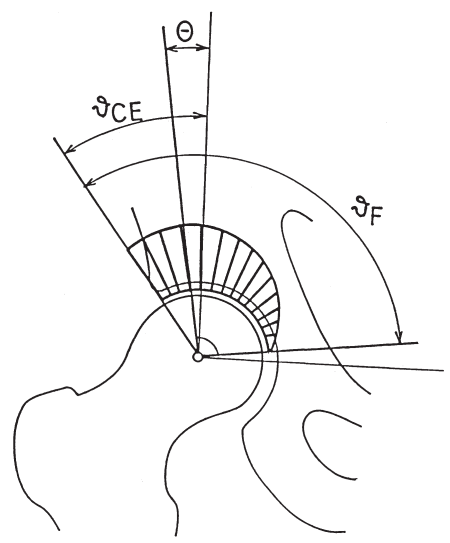

FIGURE 3 Schematic representation of the functional angle of the weight bearing area $\vartheta_{\mathrm{F}}$ and the spherical coordinate of the pole $\Theta$.

Since $\mathbf{R}$ is limited to the frontal plane, the pole of stress distribution also lies in the frontal plane and is therefore determined only by one spherical coordinate, i.e. by the angle $\Theta$ (Fig. 3). The value of $\Theta$ (Fig. 3) is determined numerically from Eq. (15) using the Newton iteration method. If the pole of stress distribution is located within the weight bearing area, the location of the peak contact stress $\left(p_{\max }\right)$ coincides with the location of the pole $\left(p_{\max }=p_{o}\right)$. When the stress pole lies outside the weight bearing area, the peak contact stress is located at the point on the weight bearing surface, which is closest to the pole [19].

In the following, we introduce also the functional angle of the weight bearing area $\vartheta_{\mathrm{F}}$ :

$$
\vartheta_{\mathrm{F}}=\pi / 2+\vartheta_{\mathrm{CE}}-\Theta,
$$

which is equal to the size of the weight bearing area divided by $2 r^{2}$ (Fig. 3).

\section{RESULTS AND CONCLUSIONS}

In order to illustrate the ability of the presented mathematical models and the corresponding computer program HIPSTRESS (written in TURBO PASCAL and in VISUAL BASIC), to simulate the effect of the femoral and pelvic shape on the contact stress distribution in the hip joint, Fig. 4 shows the dependence of the magnitude $(R)$ and the direction $\left(\vartheta_{\mathrm{R}}\right)$ of the hip joint contact force and of the peak stress in the hip joint $\left(p_{\max }\right)$ on the variation of the interhip distance $(\delta \ell)$. It can be seen in Fig. 4 that the peak stress in the human hip joint articular surface $p_{\max }$ has a minimal value for the pelvis with a small interhip distance and a maximal value for the pelvis with a large interhip distance. It can be also seen in Fig. 4 that the change of the interhip distance $(\delta \ell)$ considerably influences the magnitude of the resultant hip joint force $(R)$, however, it has nearly no effect on the angle of the inclination of this force with respect to the sagittal plane of
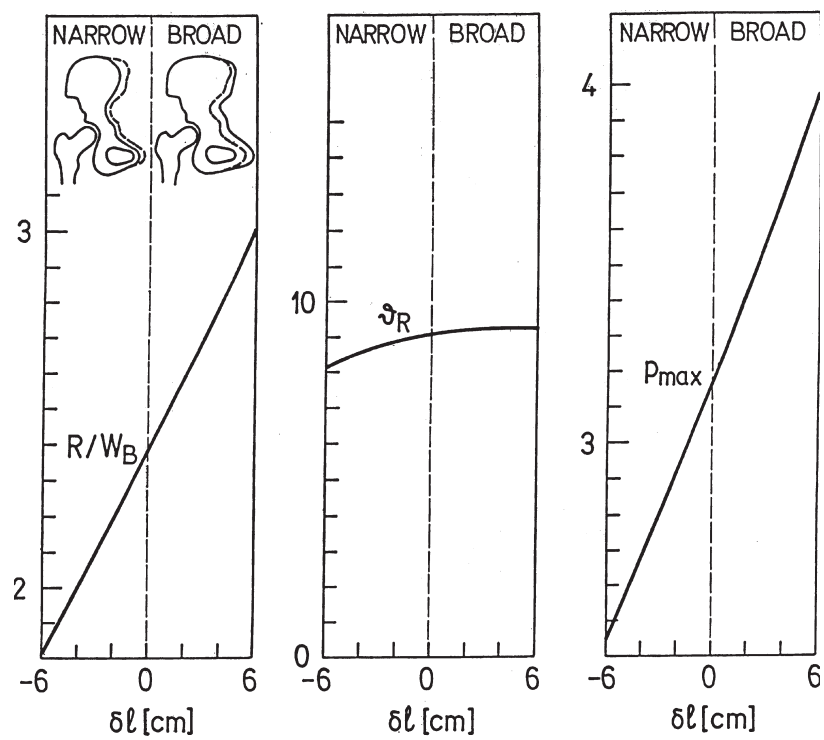

FIGURE 4 The magnitude of the resultant hip joint force $(R)$, the angle of the inclination of the hip joint resultant force with respect to the sagittal plane of the body $\left(\vartheta_{\mathrm{R}}\right)$ and the peak stress in the articular surface of the human hip joint $\left(p_{\max }\right)$ as functions of the change of the reference interhip distance $(\delta \ell)$. Parameters used in calculations are: $r=2.5 \mathrm{~cm}$, $\vartheta_{\mathrm{CE}}=29^{\circ}$ and body weight $W_{\mathrm{B}}=800 \mathrm{~N}$.

the body $\left(\vartheta_{\mathrm{R}}\right)$. This is reflected also in the peak stress in the articular surface of the hip joint $\left(p_{\max }\right)$ which increases proportionally to $R$. Namely, $p_{\max }$ is for constant $\vartheta_{\mathrm{R}}$ directly proportional to $R$ (see Eqs. (13) and (16)). The reason that $\vartheta_{\mathrm{R}}$ remains nearly constant upon the change of the interhip distance $\ell$ is that the relative position of the hip abductor muscle origin and the insertions points remain unchanged upon the variation of the interhip distance $\ell$, as they are located on the lateral side of the pelvis and on the femur. On the basis of the results presented in Fig. 4, we would like to emphasize that the pelvic shape with a large interhip distance is most probably unfavorable regarding the degenerative changes in the hip joint because of the increased stress in the articular surface of the joint.

In the following, the peak stress in the hip joint $\left(p_{\max }\right)$ is estimated from the standard anteroposterior roentgenographs for a group of normal hips and for a group of dysplastic hips. Dysplasia of the hip refers to mechanical deformations and deviations in the size and shape or mutual proportions between the upper part of the femur and the acetabulum [8]. The dysplastic hip can be diagnosed according to anatomical changes in the hip that are visible in the radiograph, as for example the presence of osteophytes or according to the shape and density of the trabecular net in the femur $[8,21,22]$. The main radiographic parameter that is used for assessment of the hip dysplasia is the centre-edge angle $\vartheta_{\mathrm{CE}}[21,22]$. The size of $\vartheta_{\mathrm{CE}}$ gives the numerical value of the lateral coverage of the femoral head (Fig. 2). The range from 20 to $25^{\circ}$ is considered to be a lower limit for normal hips, while a value below $20^{\circ}$ is pathognomonic for hip dysplasia [22]. However, it was suggested that beside $\vartheta_{\mathrm{CE}}$, the radius of 


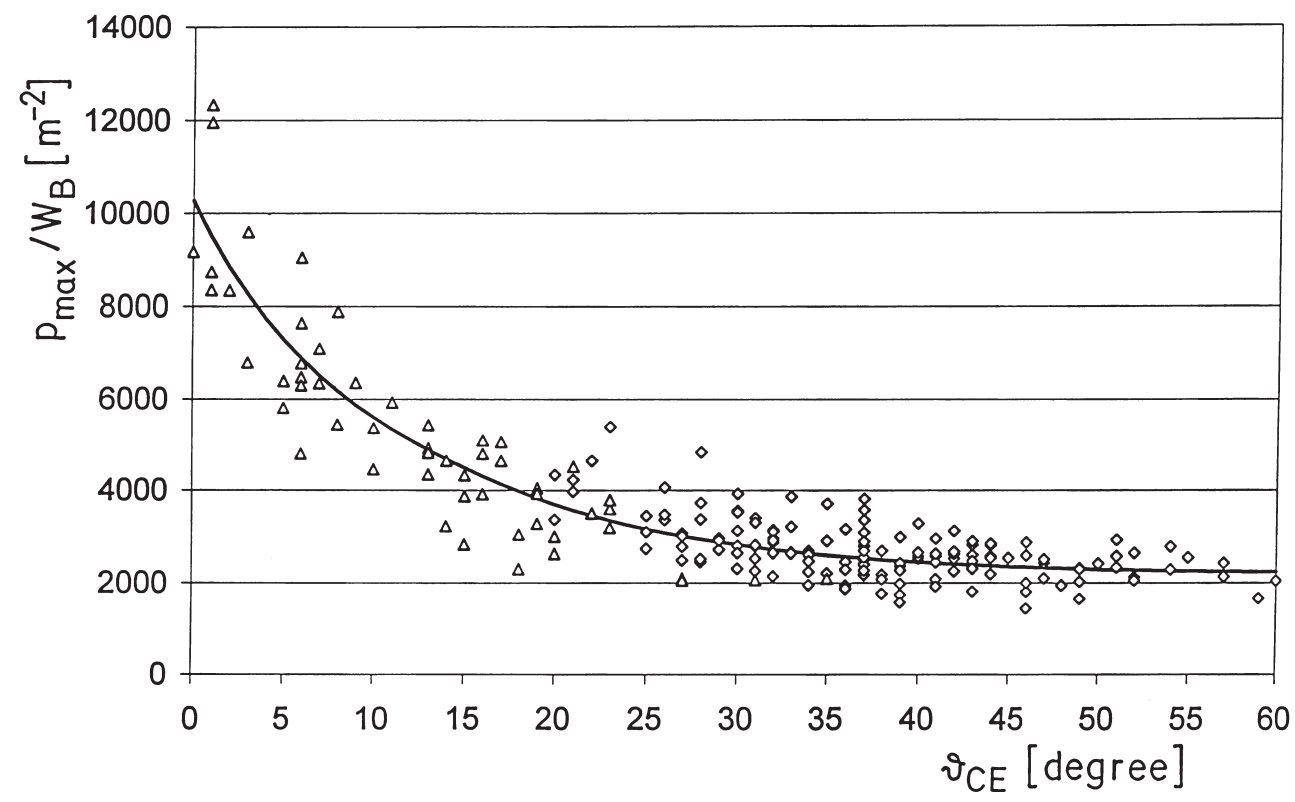

FIGURE 5 The correlation between the normalized peak contact stress $p_{\max } / W_{\mathrm{B}}$ and the centre-edge angle $\vartheta_{\mathrm{CE}}$. The values for the normal hips are denoted by the symbol $\diamond$ and the values for the dysplastic hips are denoted by the symbol $\Delta$.

the femoral head should also be taken into account in assessment of hip dysplasia [22]. It was shown that in the normal hips $\vartheta_{\mathrm{CE}}$ correlates with the femoral head radius. Hips with large heads were found to have smaller $\vartheta_{\mathrm{CE}}$ [22]. The radiographic parameters used in assessment of hip dysplasia were actually introduced to estimate the physical quantities such as hip joint contact stress distribution and size of the weight bearing area [21]. The direct estimation of physical quantities such as the peak contact stress in the hip joint $p_{\max }$ and the functional angle $\vartheta_{\mathrm{F}}$, could be important in assessment of hip dysplasia.

In this work, a group of dysplastic hips and a group of normal hips were examined with respect to the normalized peak contact stress $p_{\max } / W_{\mathrm{B}}$ and with respect to the functional angle of the weight bearing area $\vartheta_{\mathrm{F}}$. The correlation between the parameters $p_{\max } / W_{\mathrm{B}}$ (and $\vartheta_{\mathrm{F}}$ ) and the centre-edge angle $\vartheta_{\mathrm{CE}}$ was studied. The standard anteroposterior radiographs of dysplastic and normal hips were taken from the medical records of the Department of Orthopaedic Surgery and Department of Traumatology, University Medical Centre, Ljubljana. The group of dysplastic hips consists of 20 subjects with unilateral dysplasia and 18 subjects with bilateral dysplasia. In total, we have 56 dysplastic hips. In this group, 9 hips belong to males and 47 belong to females, 32 hips are right and 24 are left. The normal hips belong to 146 persons who were subject to the $\mathrm{X}$-ray examination of the pelvic region for reasons other than degenerative diseases of the hip joint. The radiographs showed no signs of the hip pathology. The contours of the bony structures in each anteroposterior radiograph were put into digital form and then measurements of the geometrical parameters (Fig. 2) were performed. The geometrical parameters needed for determination of the resultant hip force (Fig. 2) are the interhip distance $(\ell)$, the pelvic height $(H)$, the pelvic width $(C)$ and the coordinates of the effective muscle attachment point on the greater trochanter $(T)$. Digitization of the contours from the radiographs and determination of the geometrical parameters were performed by using a computer program HIJOMO as described in detail elsewhere [6,18]. The same program HIJOMO was also used to determine the centre-edge angle $\vartheta_{\mathrm{CE}}$ and the femoral head radius $r$ needed for calculation of the parameter $p_{\max } / W_{\mathrm{B}}$ and the functional angle $\vartheta_{\mathrm{F}}$.

The correlation between the centre-edge angle $\vartheta_{\mathrm{CE}}$ and the normalized peak contact stress $p_{\max } / W_{\mathrm{B}}$ is shown in Fig. 5. The shape of the numerically obtained fitting curve is consistent with the above-described mathematical model of the contact stress distribution in the hip joint. Low $\vartheta_{\mathrm{CE}}$ correlates with high $p_{\max } / W_{\mathrm{B}}$ and vice versa. Scattering of the data in Fig. 5 shows that in determining the peak contact stress, the geometrical parameters other than $\vartheta_{\mathrm{CE}}$ angle are also important. For example, in two hips with approximately the same $\vartheta_{\mathrm{CE}}\left(6^{\circ}\right)$ the normalized peak stress was shown to differ by about $4000 \mathrm{~m}^{-2}$. The influence of the geometrical parameters other than $\vartheta_{\mathrm{CE}}$ on the value of $p_{\max } / W_{\mathrm{B}}$ is larger for smaller $\vartheta_{\mathrm{CE}}$ angles.

Figure 6 shows the correlation between the center-edge angle $\vartheta_{\mathrm{CE}}$ and the functional angle of the weight bearing $\vartheta_{\mathrm{F}}$. In Fig. 6, we can see a positive correlation between $\vartheta_{\mathrm{F}}$ and $\vartheta_{\mathrm{CE}}$; high values of $\vartheta_{\mathrm{CE}}$ correlate with high values of $\vartheta_{\mathrm{F}}$. The influence of the geometrical parameters other than $\vartheta_{\mathrm{CE}}$ on the functional angle $\vartheta_{\mathrm{F}}$ (reflected in the scattering) is low.

To characterize the role of the parameters $p_{\max } / W_{\mathrm{B}}$ and $\vartheta_{\mathrm{F}}$ in the assessment of hip dysplasia, statistical significance of the differences in the parameters $p_{\max } / W_{\mathrm{B}}$ and $\vartheta_{\mathrm{F}}$ between the normal and dysplastic hips 


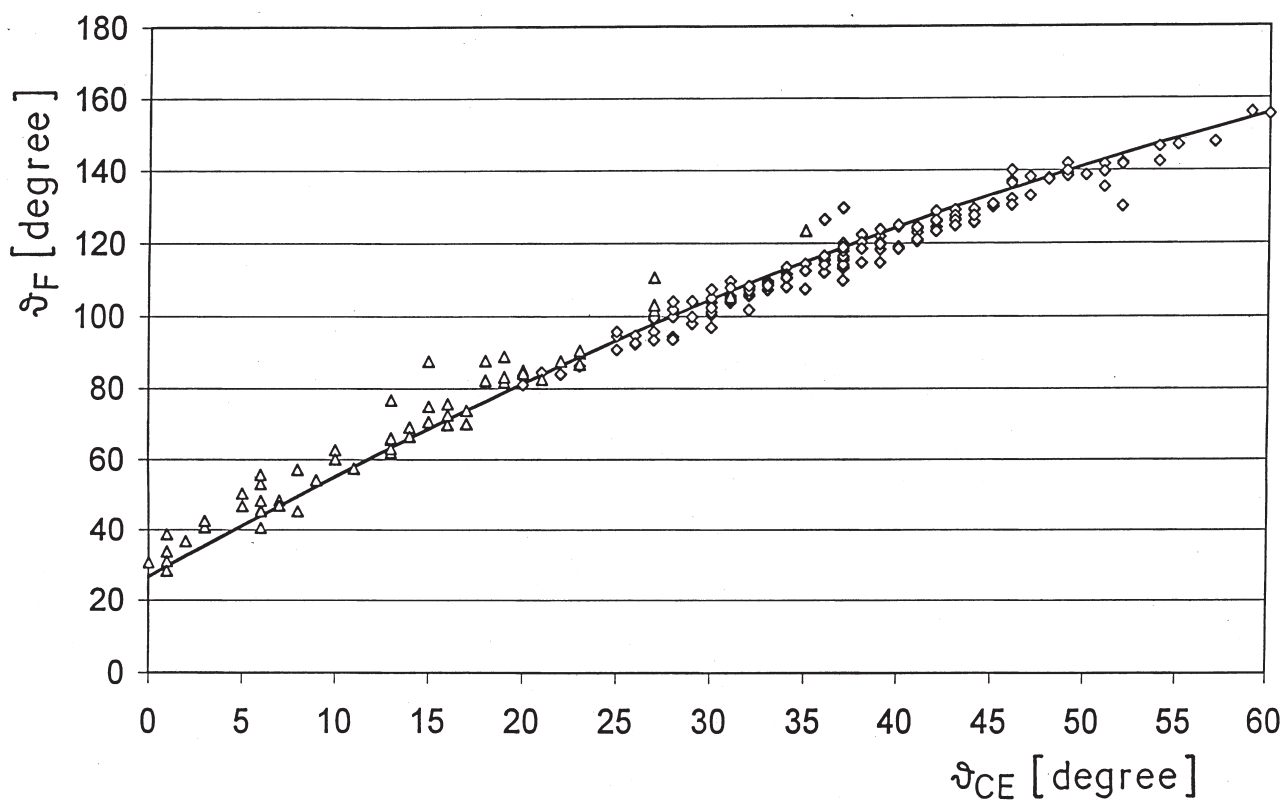

FIGURE 6 The correlation between the functional angle of the weight bearing $\vartheta_{\mathrm{F}}$ and the centre-edge angle $\vartheta_{\mathrm{CE}}$. The values for the normal hips are denoted by the symbol $\diamond$ and the values for the dysplastic hips are denoted by the symbol $\Delta$.

was calculated by the two-tailed pooled $t$-test [23]. The average values of the sets of the data of the particular biomechanical parameter and the results of the $t$-test are given in Table III. The null hypothesis [8] assuming equal average values of $p_{\max } / W_{\mathrm{B}}$ and $\vartheta_{\mathrm{F}}$ in normal and dysplastic hips is rejected at the level lower than 0.001. It can be therefore concluded that parameters $p_{\max } / W_{\mathrm{B}}$ and $\vartheta_{\mathrm{F}}$ are appropriate biomechanical parameters for the assessment of hip dysplasia.

The normalized peak contact stress $p_{\max } / W_{\mathrm{B}}$ and the size of the weight bearing area (which is proportional to $\vartheta_{\mathrm{F}}$ ) have been estimated from the anteroposterior radiographs already before $[20,22]$. However, in these studies the hip joint contact force was determined by using the simple mathematical models, which consider only a single effective muscle group (the hip abductors) and very few direct musculoskeletal anatomical data. Consequently, the variation of the model parameters by means of the variation of the geometrical parameters of the femoral and pelvic shape is strongly limited [24]. More accurate estimation of the hip joint contact force requires three-dimensional muscle model [11,24]. In this work, the hip joint contact force is calculated by using a threedimensional mathematical model that includes nine effective muscles (Table I). The coordinates of the muscle pelvic and femoral attachment points are taken from Dostal and Andrews [10].

TABLE III The average values of $p_{\max } / W_{\mathrm{B}}$ and $\vartheta_{\mathrm{F}}$ in the normal and dysplastic hips

\begin{tabular}{lccc}
\hline Parameter & Normal & Dysplastic & Difference $(P)$ \\
\hline$p_{\max } / W_{\mathrm{B}}\left(\mathrm{m}^{-2}\right)$ & 2692 & 5274 & $<0.001$ \\
$\vartheta_{\mathrm{F}}($ degree $)$ & 117 & 66.8 & $<0.001$ \\
\hline
\end{tabular}

In conclusion, we describe a method for determination of the hip joint contact force, the peak contact stress and the functional angle of the weight bearing area from the standard anteroposterior radiographs by using the mathematical model. A population study indicated that by applying the described method the average value of the normalized peak contact stress $p_{\max } / W_{\mathrm{B}}$ and the average value of the functional angle of the weight bearing $\vartheta_{\mathrm{F}}$ are in dysplastic hips twice as large as the respective quantities in normal hips. It was also indicated that the pelvic shape with a large interhip distance is most probably unfavorable regarding the degenerative changes in the hip joint because of the increased stress in the articular surface of the joint.

\section{Acknowledgements}

The authors thank R. Vengust, S. Herman, O. Zupanc, B. Kersnič, M. Drobnič, B. Mavčič and B. Pompe for gathering the radiographs from the archives and determining the profiles of the hip and pelvis. Equations (13) and (14) are cited from the paper Ipavec et al. [19] where the typographical error in Eqs. (5) and (6) appeared (the symbol \pm should be replaced by $\mp$ ). The computer program HIPSTRESS for calculation of the hip joint contact force, contact stress distribution and functional angle of the weight bearing area can be obtained from the authors by e-mail free of charge.

\section{References}

[1] Mow, V.C., Proctor, C.S. and Kelly, M.A. (1989) "Biomechanics of articular cartilage", In: Nordin, M. and Frankel, V., eds, Basic Biomechanics of the Musculoskeletal System (Lea and Febiger, London), pp 31-58. 
[2] Legal, H., Reinecke, M. and Ruder, H. (1980) "Zur biostatischen Analyse des Hüftgelenks III", Zeitschrift für Orthopadie und ihre Grenzgebiete 118, 804-815.

[3] Hadley, N.A., Brown, T.D. and Weinstein, S.L. (1990) "The effects of contact pressure elevations and aseptic necrosis on the long-term clinical outcome of congenital hip dislocation", Journal of Orthopaedic Research 8, 504-513.

[4] Maxian, T.A., Brown, T.D. and Weinstein, S.L. (1995) "Chronic stress tolerance levels for human articular cartilage: two nonuniform contact models applied to long term follow-up of CDH", Journal of Biomechanics 28, 159-166.

[5] Brand, R.A. (1997) "Hip osteotomies. A biomechanical consideration", Journal of the American Academy of Orthopaedic Surgeons 5, 282-291.

[6] Iglič, A., Daniel, M., Kralj-Iglič, V., Antolič, V. and Jaklič, A. (2001) "Peak hip-joint contact stress in male and female populations", Journal of Musculoskeletal Research 5, 17-21.

[7] Kersnič, B., Iglič, A., Kralj-Iglič, V., Srakar, F. and Antolič, V. (1997) "Incresed incidence of arthrosis in women could be related to femoral and pelvic shape", Archives of Orthopaedics and Trauma Surgery 116, 345-347.

[8] Durnim, C.W., Ganz, R. and Klaise, K. (1991) "The acetabular rim syndrome- a clinical presentation of dysplasia of the hip", Journal of Bone and Joint Surgery 73B, 423-429.

[9] Iglič, A., Srakar, F. and Antolič, V. (1993) "Influence of the pelvic shape on the biomechanical status of the hip", Clinical Biomechanics 8, 233-234.

[10] Dostal, W.F. and Andrews, J.G. (1981) "A three dimensional biomechanical model of the hip musculature", Journal of Biomechanics 14, 803-812.

[11] Johnston, R.C., Brand, R.A. and Crowninshield, R.D. (1979) "Reconstruction of the hip", Journal of Bone and Joint Surgery 61A, 639-652.

[12] Mcleish, R.D. and Charnley, J. (1970) "Abduction forces in the onelegged stance", Journal of Biomechanics 3, 191-209.

[13] Clauser, C.E., McConville, J.T. and Young, J.W. (1969) Weight, Volume and Centre of Mass of Segments of the Human Body (Aerospace Medical Research Laboratory, Aerospace Medical Division, WPAF Base, OH (USA)).
[14] Kralj, A. (1969) "Optimum coordination and selection of muscles for functional electrical stimulation". In Proceedings of the 8th ICMBE, Chicago, p. 7.

[15] Maček-Lebar, A., Iglič, A., Antolič, V. and Srakar, F. (1993) "Distribution of the hip abductor muscle forces in one-legged stance", Journal of Slovene Medical Society 62, 231-232.

[16] Dixon, L.C. (1970) Nonlinear Optimization (The English Universities Press, Bell and Bain, Glasgow).

[17] Antolič, V., Kralj-Iglič, V., Iglič, A. and Srakar, F. (1993) "LeggCalvé-Perthers disease-stress distribution on the hip joint articular surface after varisation osteotomy", In: Held, K.D., Brebbia, C.A., Ciskowski, R.D. and Power, H., eds, Computational Biomedicine (Computational Mechanics Publications, Southampton, Boston), pp 255-262.

[18] Zupanc, O., Antolič, V., Iglič, A., Jaklič, A., Kralj-Iglič, V., Stare, J. and Vengust, R. (2001) "Assessment of the contact stress in the hip joint after operative treatment of severe slipped capital femoral epiphysis", International Orthopaedics (SICOT) 25, 9-12.

[19] Ipavec, M., Brand, R.A., Pedersen, D.R., Mavčič, B. and KraljIglič, V. (1999) "Mathematical modelling of stress in the hip during gait", Journal of Biomechanics 32, 1229-1235.

[20] Brinckmann, P., Frobin, W. and Hierholzer, E. (1981) "Stress on the articular surface of the hip joint in healthy adults and persons with idiopathic osteoarthrosis of the hip joint", Journal of Biomechanics 14, 149-156.

[21] Pauwels, F. (1976) Biomechanics of the Normal and Diseased Hip (Springer, Berlin), pp 1-22.

[22] Legal, H. (1987) "Introduction to the biomechanics of the hip", In: Tönnis, D., ed, Congenital Dysplasia and Dyslocation of the Hip (Springer, Berlin), pp 26-57.

[23] Duncan, R.B., Knapp, R.B. and Miller, M.C. (1977) Introductory Biostatistics for the Health Sciences (Wiley, New York), pp 21-119.

[24] Brand, R.A. and Pedersen, D.R. (1984) "Computer modeling of surgery and a consideration of the mechanical effects of proximal femoral osteotomies". In Proc. 12th Open Scientific Meeting of the Hip Society (ed. R.B. Welch), Mosby, St. Louis, pp. 193-210. 
Copyright $\odot 2003$ EBSCO Publishing 